\title{
Numerical pressure transient analysis for unfilled-caved carbonate reservoirs based on Stokes-Darcy coupled theory
}

\author{
Yizhao Wan ${ }^{\mathrm{a}, \mathrm{b}, *}$, Yuewu Liu ${ }^{\mathrm{c}}$, Nengyou Wu ${ }^{\text {a,b }}$ \\ ${ }^{a}$ The Key Laboratory of Gas Hydrate, Ministry of Natural Resources of the People's Republic of China, Qingdao Institute of Marine Geology, Qingdao, 266071, China \\ ${ }^{\mathrm{b}}$ Laboratory for Marine Mineral Resources, Qingdao National Laboratory for Marine Science and Technology, Qingdao, 266071, China \\ ${ }^{\mathrm{c}}$ Institute of Mechanics, Chinese Academy of Science, Beijing, 100019, China
}

\section{A R T I C L E I N F O}

\section{Keywords:}

Carbonate reservoir

Pressure transient behavior

Stokes-Darcy coupled

Flow in porous media

Numerical simulation

\begin{abstract}
A B S T R A C T
Caved carbonate reservoirs are very special because of the strong heterogeneity. The pressure transient behavior of the caved carbonate reservoirs is quite different from the conventional homogeneous or dual medium reservoirs because of the presence of large-scale cavities. There are two types of cavities: filled and unfilled, which dominated the production of the reservoirs. Fluid flow in the unfilled cavity should be described by Stokes' equation rather than Darcy's law. It is needed to better understand the role of the unfilled cavities plays in the pressure transient analysis. The objective of this work is to analyze the pressure transient behavior of the unfilled cavities. A coupled Stokes-Darcy pressure transient model is developed and the finite element method is applied in the solutions of the mathematical models. Then, the numerical pressure transient model is used in the analysis of two typical cases: a well drilled into the unfilled cavity (WIC) and a well not drilled into the unfilled cavity (WOC). The type curves of the WIC model indicate that flow in the unfilled cavity is an oscillated pressure-drop rather than a radial flow. The unfilled cavity that the well drilled into would be considered as an enlarged wellbore which is equivalent to a negative skin factor, as a consequence the wellbore storage coefficient will increase. Main characteristics of type curves for WOC model are the valley on the pressure derivative. A cavity with a larger size and smaller distance from the wellbore would give rise to a deeper valley. Comparative results indicate that unfilled cavities described by the Stokes' equation are not the limit of the filled cavities with extremely large mobility, which was predicted by previous work.
\end{abstract}

\section{Introduction}

The naturally fractured-vuggy carbonate reservoirs are ubiquitous in the world (Li et al., 2018). The pores in this kind of reservoirs are comprised of the matrix, fractures, and vugs. The size of vugs in naturally fractured reservoirs is centimeters or millimeters in diameter (Wu et al., 2011; Huang et al., 2013). However, in the Tarim oil field in China, large-scaled vugs also named cavities of several meters or even tens of meters are found in the formation (Zhao et al., 2014), which can be identified by "beads-like" reflection in the seismic profile (Xiao et al., 2010). The existence of cavities is also supported by the drilling breaks and mud leakage (Zhu et al., 2015; Li et al., 2016), and the cavities with drilling breaks are unfilled. Except for the unfilled cavities, there are some cavities filling with sand, gravel, and mud (Popov et al., 2009; Hu et al., 2014; Wan et al., 2018a). We call the reservoirs composed of the matrix and cavities caved carbonate reservoirs. It is notable that the cavities in this context indicate the large-scale entities in the reservoirs which should be differentiated from the vugs.

In order to understand the hydraulic behaviors of the caved reservoirs, pressure transient analysis is widely used (Wan et al., 2016, 2018b; Wan and Liu, 2016), which can obtain the reservoirs' information through the pressure behavior vs. time at the wellbore. However, the pressure transient analysis for the caved carbonate reservoirs is difficult because of the large-scale cavities. For the filled cavities, the continuous theories can be applied and the flow in the filled cavities obey Darcy's law (Popov et al., 2009). Some dual media models are established to analyze the pressure transient behavior of caved carbonate (Djatmiko Hansamuitet al., 2010; Gomez et al., 2014). In these models, the filled cavities are considered as the secondary porosity and fluid transfer from the cavities to the matrix described by a transient

\footnotetext{
* Corresponding author. The Key Laboratory of Gas Hydrate, Ministry of Natural Resources of the People's Republic of China, Qingdao Institute of Marine Geology, Qingdao, 266071, China

E-mail address: wanyizhao@mail.cgs.gov.cn (Y. Wan).
} 


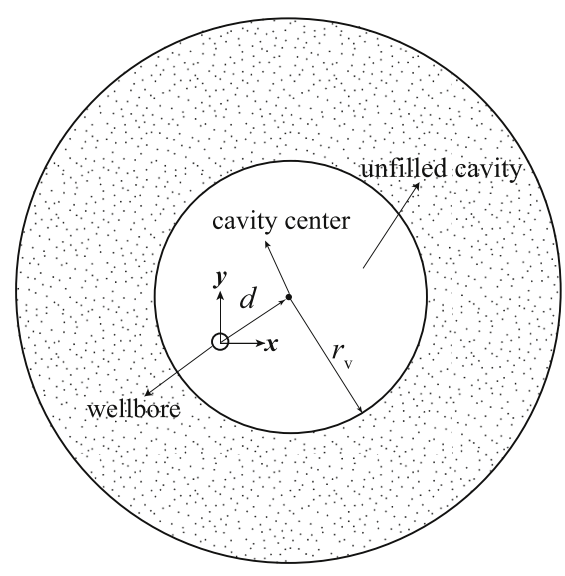

(a) WIC model

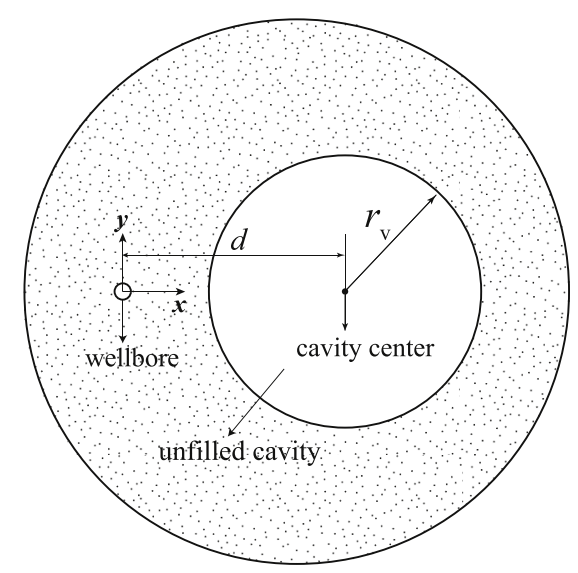

(b) WOC model

Fig. 1. Physical concept model (modified from Wan et al., 2018a).

function. Taking fractures into account, some researchers developed triple porosity models which consider the fluid transformation from matrix and cavities to fractures (Wu et al., 2007). Nevertheless, the dual/triple media models essentially assume that the cavities are distributed uniformly in the whole reservoirs, which doesn't correspond with the geological characterization of caved carbonate reservoirs. Wan et al. (2018a), pointed out that the dual media models are not appropriate in the caved carbonate reservoirs. They proved that the valley on the pressure derivative curve is dominated by the cavities rather than the dual media effect. The dual media models would overestimate the storage capability of the cavities. Thus, discrete models are developed to analyze the pressure transient behavior of filled-caved carbonate reservoirs. In these models, the cavities are described explicitly by Darcy flow zones with an extremely high permeability and porosity. The regions beyond the cavities are treated as single-porosity media with lower permeability or dual-porosity media (Gao et al., 2016). As much research pointed out (Zhang et al., 2012; Hu et al., 2014; Wan et al., 2018a), the pressure transient behavior is dominated by the cavities. Whether a well is drilled into a cavity has a great effect on the pressure transient behavior, so the analytical or numerical models of a well in the cavity and not in the cavities are proposed respectively (Zhang et al., 2009; Gao et al., 2016; Wan et al., 2018a). These models have been successfully implemented in the interpretation of field data.

The situation in the case of unfilled cavities is more complicated and quite different from filled cavities. As unfilled, the cavities can't be considered as porous media, and naturally, Darcy' law is inapplicable. In the previous work, the Stokes equation is used to describe the fluid flow in the unfilled cavities, thus the core of the fluid flow becomes a StokesDarcy coupled problem (Arbogast and Gomez, 2009; Gulbransen et al., 2010; Huang et al., 2011). In this problem, the boundary condition of the interface between a Stokes flow region and a Darcy flow region is the key. It is difficult because the Stokes equation is second-order but the Darcy's law is no-order, which leads to discontinuity on the interface (Mikelic and Jäger, 2000; Deng and Martinez, 2005). The obvious condition on the interface is the continuity of the fluid flow in the normal direction and the fluid flow in the tangential direction is ignored. However, this condition cannot describe the true behavior of the fluid flow through the interface (Mikelic and Jäger, 2000). Beavers and Joseph (Beavers and Joseph, 1967; Beavers et al., 1970) proposed a condition that the difference of the velocity in the tangential direction between the Stokes' zone and Darcy's zone is proportional to the shear rate of the free fluid (Mikelic and Jäger, 2000). Saffman (1971) modified the condition by ignoring the Darcy's velocity. The Beavers-Joseph-Saffman boundary condition is the most commonly used one for the Stokes-Darcy coupled flow.
The method that divides the whole problem into two domains composed of free fluid flow region and Darcy's flow region and introduces the boundary on the interface is called the dual domain method (Yao et al., 2010; Deng and Martinez, 2005). Besides, researchers combined the Stokes' equation and Darcy's law and developed a uniform governing equation for both the Stokes and Darcy flow region. Thus the free-fluid flow region and Darcy flow region are combined to a composite domain. A parameter in the uniform governing equation is proposed to discriminate the two regions. This method is called the single domain method (Deng and Martinez, 2005; Huang, 2012). Since the two domains are collapsed into one composite domain, there is no need the condition on the interface any more. This one-domain method is validated by Deng et al. (Deng and Martinez, 2005).

Peng et al. (2009),' s research shows that the saturation and pressure distribution of a Stokes-Darcy model and conventional Darcy model is very similar in the Darcy-flow region. However, in the Stokes equation governed region, velocities of the Stokes-Darcy model are more accurate. The results of the Stokes-Darcy coupled simulation also indicate that the pressure spreads very quickly in cavities, so the cavities can be treated as equipotential bodies (Gao et al., 2016). Chen et al. (2016), developed a pressure-transient model for the well is not drilled into the cavity which is treated as an equipotential body, while Liu et al. (Liu and Wang, 2012), developed a simplified radial composite for the well is drilled into a cavity. Some other researchers also proposed similar models accounting for dual porosity media in the Darcy-flow region (Xing et al., 2018).

It should be noticed that fractures are also very common in the caved carbonate reservoirs. The large-scale fractures have strong effects on the pressure behavior (Wan et al., 2019; Jiang et al., 2019; Raghavan and Chen, 2019), and the behavior of the cavities may be covered by the fractures. Since we want to focus on the behavior of the cavities especially the unfilled ones, the large-scale fractures are ignored. The behavior of large numbers of small fractures can be described by dual-media theory, and a valley on the pressure derivative curve will be observed. However, in the fractured-caved carbonated reservoir, the interporosity flow coefficient $\lambda$ is quite small because the permeability of the matrix is extremely low. Consequently, the valley caused by the dual-media effect appears at very late time and always cannot be detected by the well-test which is last for no more than 200 hours. Thus, we assume that the fractures are merged in the matrix and an equivalent "matrix" with an equivalent permeability is presented.

The cavities can be treated as equipotential bodies because the time of pressure spreading in cavities is very small which means the pressure in the cavity is almost the same. However, in the pressure transient analysis, the bottom-hole pressure is the function of time and the 
pressure derivative can capture physical processes in a very small-time scale especially in the early time of the logarithmic coordinates. Hence, the equipotential assumption may be fine in the numerical simulation, but miss some important behaviors in the pressure transient analysis. The objective of this study is to analyze the pressure transient behavior of the unfilled cavities directly based on the Stokes-Darcy coupled model.

\section{Physical concept and mathematical model}

\subsection{Physical concept model}

As analyzed above, the pressure behaviors of a well drilled into the cavity and not into the cavity which are abbreviated as WIC and WOC respectively, are quite different. Thus, these two models are considered in this study. Fig. 1 shows the physical model, in which the cavity is represented by a circle with a radius $r_{v}$. The distance from the wellbore to the center of the cavity is $d$. Moreover, some other assumptions are made as followings:

- The cavity is unfilled and Stokes' equation is applied in the description of fluid flow. The region beyond the cavity is matrix porous media and the Darcy's law is applied.

- The cavity is full of fluid and the fluid in the cavity is incompressible, while the fluid in the pores is lightly compressible and the compressibility coefficient is constant.

- The wellbore storage effect are considered, but skin, gravity, and capillary effect are neglected.

\subsection{Mathematical model}

The mass conservation for lightly compressible fluid flow in the matrix is:

$\varphi c_{\mathrm{t}} \frac{\partial p_{\mathrm{m}}}{\partial t}+\nabla \boldsymbol{u}_{\mathrm{m}}=0$

where $\varphi$ is the porosity of the matrix, $p_{\mathrm{m}}$ is the pressure in the matrix, $\boldsymbol{u}_{\mathrm{m}}$ is the Darcy's seepage velocity vector, and $c_{\mathrm{t}}$ is the total compressibility coefficient.

The flow in the matrix region obey Darcy's law, and the momentum conservation equation is:

$\boldsymbol{u}_{\mathrm{m}}=-\frac{k_{\mathrm{m}}}{\mu} \nabla p_{\mathrm{m}}$

where $k_{\mathrm{m}}$ is the permeability of the matrix, $\mu$ is the viscosity of the fluid.

The mass conservation for the fluid in the cavity region is:

$\nabla \boldsymbol{u}_{\mathrm{c}}=0$

where $\boldsymbol{u}_{\mathrm{c}}$ is the fluid flow velocity vector in the cavity.

The Stokes' equation is applied in the fluid flow description in the unfilled cavity:

$\rho \frac{\partial \boldsymbol{u}_{\mathrm{c}}}{\partial t}=-\nabla p_{\mathrm{c}}+\mu \nabla^{2} \boldsymbol{u}_{\mathrm{c}}$

where $\rho$ is the fluid density, $p_{c}$ is the pressure in the cavity.

Using the single domain method, the mass conservation equations (1) and (3) can be written as:

$(1-D) \varphi c_{\mathrm{t}} \frac{\partial p}{\partial t}+\nabla \boldsymbol{u}=0$

where $D$ is a model control coefficient, and in the cavity region $D=1$ equation (5) reduces to equation (3), otherwise in the matrix region $D=$ 0 equation (5) reduces to equation (1).

In the same way, the momentum conservation equation (2) and equation (4) can be written as:

$D \rho \frac{\partial \boldsymbol{u}}{\partial t}=(D-1) \frac{\mu}{k_{\mathrm{m}}} \boldsymbol{u}+D \mu \nabla^{2} \boldsymbol{u}-\nabla p$

and in the cavity region $D=1$, equation (6) reduces to equation (4); in the matrix region $D=0$, equation (6) reduces to equation (2).

Then the governing equation of two-dimension for the unfilledcavity model is:

$\left\{\begin{array}{c}(1-D) \varphi c_{\mathrm{t}} \frac{\partial p}{\partial t}+\frac{\partial u}{\partial x}+\frac{\partial v}{\partial y}=0 \\ D \rho \frac{\partial u}{\partial t}=(D-1) \frac{\mu}{k} u+D \mu\left(\frac{\partial^{2} u}{\partial x^{2}}+\frac{\partial^{2} u}{\partial y^{2}}\right)-\frac{\partial p}{\partial x} \\ D \rho \frac{\partial v}{\partial t}=(D-1) \frac{\mu}{k} v+D \mu\left(\frac{\partial^{2} v}{\partial x^{2}}+\frac{\partial^{2} v}{\partial y^{2}}\right)-\frac{\partial p}{\partial y}\end{array}\right.$

where $u$ and $v$ are the fluid flow velocities in the $x$ and $y$ directions, respectively.

The initial condition is that the pressure maintains the initial pressure of the reservoir, and the velocity is zero, that is:

$u=0, v=0, p=p_{\mathrm{i}}$

where $p_{\mathrm{i}}$ is the initial reservoir pressure.

The inner boundary condition is the mass conservation in the wellbore boundary, that is:

$\left.2 \pi r_{\mathrm{w}} u_{\mathrm{n}}\right|_{\Gamma_{\mathrm{i}}}=q B+C \frac{d p_{\mathrm{w}}}{d t}$

where $r_{\mathrm{w}}$ is the wellbore radius, $u_{\mathrm{n}}$ is the fluid flow velocity in the direction normal to the wellbore boundary $\Gamma_{i}, q$ is the rate and assumed as constant, $B$ is the volume factor of the fluid, $C$ is the wellbore storage coefficient, $p_{\mathrm{w}}$ is the wellbore pressure.

The reservoir boundary condition is assumed to be no fluid flow boundary, that is:

$\left(\frac{\partial p}{\partial n}\right)_{\Gamma_{0}}=0$

where $\Gamma_{\mathrm{o}}$ represents the reservoir boundary, and $\mathrm{n}$ is the normal direction of $\Gamma_{0}$.

The continuous boundary condition between the interface between the cavity and the matrix is satisfied automatically because the fluid flow in the two regions is described by the same governing equation (7).

\subsection{Numerical simulation}

The Galerkin weighted residual method is used to discretize the governing equation (7). There are three equations in (7) and each of them has different physical essence, so the weight function of them should be chosen respectively. The first equation in (7) is the mass conversation formula, which represents the volume changes of fluid caused by the pressure changes. Thus, we choose the variational pressure $\delta p$ as the weight function. The second and third equation in (7) is the momentum equation in the $x$ and $y$ direction, and the Galerkin form the equation should be the virtual power balance of the fluid flow. Then, the weight function should be $\delta u$ and $\delta v$, respectively. Then, the Galerkin form equations of (7) are as follows:

$\iint\left[(1-D) \varphi c_{t} \frac{\partial p}{\partial t}+\frac{\partial u}{\partial x}+\frac{\partial \nu}{\partial y}\right] \delta p d x d y=0$

$\iint\left[\rho \frac{\partial u}{\partial t}=(D-1) \frac{\mu}{k} u+D \mu\left(\frac{\partial^{2} u}{\partial x^{2}}+\frac{\partial^{2} u}{\partial y^{2}}\right)-\frac{\partial p}{\partial x}\right] \delta u d x d y=0$ 


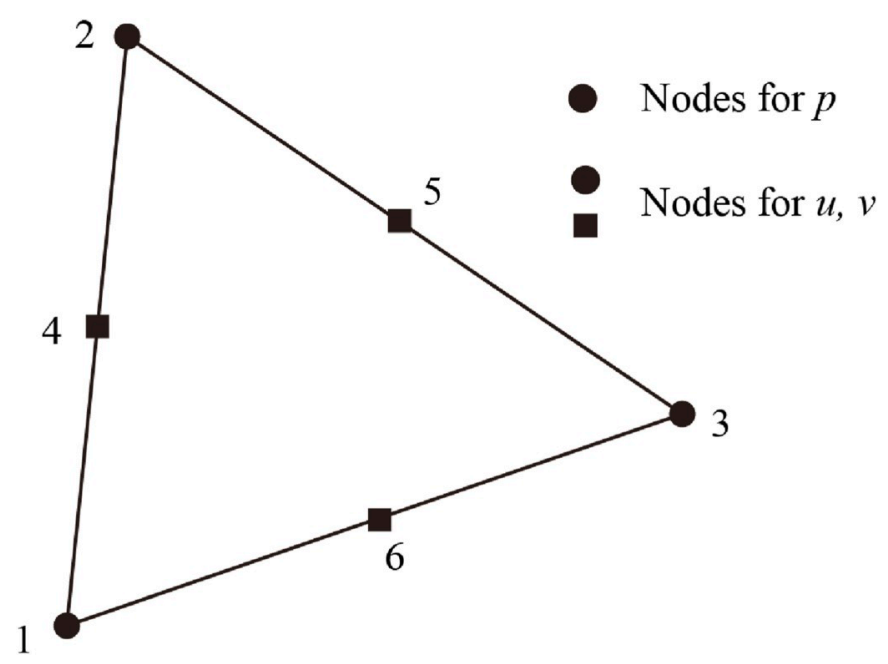

Fig. 2. The element used in the Galerkin method.

$\iint\left[D \rho \frac{\partial v}{\partial t}=(D-1) \frac{\mu}{k} v+D \mu\left(\frac{\partial^{2} v}{\partial x^{2}}+\frac{\partial^{2} v}{\partial y^{2}}\right)-\frac{\partial p}{\partial y}\right] \delta v d x d y=0$

Further, after order reducing, the above equations are derived to weak forms.

The weak form of equation (11):

$$
\begin{gathered}
\iint\left[(1-D) \varphi c_{t} \frac{\partial p}{\partial t}+\frac{\partial u}{\partial x}+\frac{\partial v}{\partial y}\right] \delta p d x d y \\
=\iint\left[(1-D) \varphi c_{t} \frac{\partial p}{\partial t} \delta p+\left(\frac{\partial(u \delta p)}{\partial x}+\frac{\partial(v \delta p)}{\partial y}\right)-\left(u \frac{\partial \delta p}{\partial x}+v \frac{\partial \delta p}{\partial y}\right)\right] d x d y \\
=\iint(1-D) \varphi c_{t} \frac{\partial p}{\partial t} \delta p d x d y+\int u_{n} \delta p d s-\iint-\left(u \frac{\partial \delta p}{\partial x}+v \frac{\partial \delta p}{\partial y}\right) d x d y=0
\end{gathered}
$$

where $s$ represents the boundary of the model.

The weak form of equation (12) is:

$$
\begin{gathered}
\iint\left[D \rho \frac{\partial u}{\partial t}-(D-1) \frac{\mu}{k} u-D \mu\left(\frac{\partial^{2} u}{\partial x^{2}}+\frac{\partial^{2} u}{\partial y^{2}}\right)+\frac{\partial p}{\partial x}\right] \delta u d x d y \\
=\iint\left(D \rho \frac{\partial u}{\partial t} \delta u-(D-1) \frac{\mu}{k} u \delta u+\frac{\partial p}{\partial x} \delta u\right) d x d y \\
-\iint D \mu\left(\frac{\partial}{\partial x}\left(\delta u \frac{\partial u}{\partial x}\right)+\frac{\partial}{\partial y}\left(\delta u \frac{\partial u}{\partial y}\right)\right) d x d y+\iint D \mu\left(\frac{\partial u}{\partial x} \frac{\partial \delta u}{\partial x}+\frac{\partial u \partial \delta u}{\partial y} \frac{\partial y}{\partial y}\right) d x d y \\
=\iint\left(D \rho \frac{\partial u}{\partial t} \delta u-(D-1) \frac{\mu}{k} u \delta u+\frac{\partial p}{\partial x} \delta u\right) d x d y+\iint D \mu\left(\frac{\partial u \partial \delta u}{\partial x} \frac{\partial u}{\partial x}+\frac{\partial \delta u}{\partial y}\right) d x d y \\
-\int D \mu \frac{\partial u}{\partial n} \delta u d s=0
\end{gathered}
$$

The weak form equation (13) are the same with equation (15) except that the variable $u$ should be $v$, so it is omitted here.

The interpolations of the variables in an element are:

$$
\begin{aligned}
& p(x, y, t)=\sum_{i=1}^{G} P_{i}(x, y) p_{i}(t) \\
& u(x, y, t)=\sum_{j=1}^{R} N_{j}(x, y) u_{j}(t) \\
& v(x, y, t)=\sum_{j=1}^{R} N_{j}(x, y) v_{j}(t)
\end{aligned}
$$

The interpolation function of variable $p$ is $P$, and the number of interpolation points is $G$. The interpolation function of variable $u, v$ is $N$, and the number of interpolation points is $R$. As can be seen from

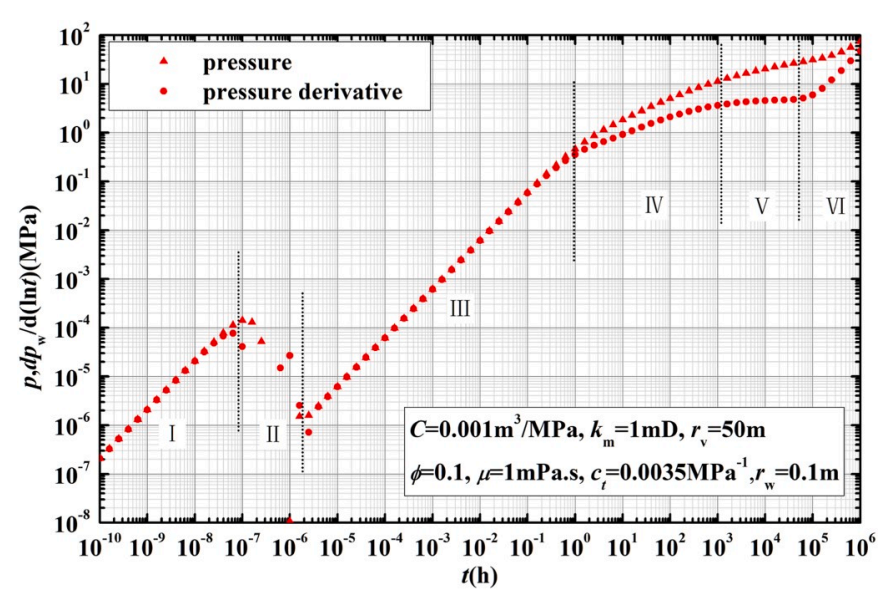

Fig. 3. The log-log plots of the bottom hole pressure and pressure derivative versus time for the WIC model.

equation (7), the derivative order of the variable $u, v$ is two, and that of the variable $p$ is one, so the order of the interpolation function $N$ for $u, v$ should be greater than that for $p$. Usually, the order of $N$ is two and that of $p$ is one. Thus, the element with six nodes in Fig. 2 should be used. As shown in Fig. 2, the interpolating points for $p$ are the vertex of the triangle and that for $u, v$ includes the midpoint of the edges besides the vertex, which indicates that $G=3, R=6$.

Substituting equation (16) into equations (14) and (15), we get:

$\sum_{m=1}^{G} \sum_{i=1}^{G} K_{i m}^{p p} \dot{p}_{i}-\sum_{m=1}^{G} \sum_{j=1}^{R} K_{j m}^{p u} u_{j}-\sum_{m=1}^{G} \sum_{j=1}^{R} K_{j m}^{p v} v_{j}=\sum_{m=1}^{G} Q_{m}$

$\sum_{m=1}^{R} \sum_{j=1}^{R} A_{j m}^{u u} \dot{u}_{j}-\sum_{m=1}^{R} \sum_{j=1}^{R} B_{j m}^{u u} u_{j}-\sum_{m=1}^{G} \sum_{i=1}^{G} C_{i m}^{u p} p_{i}=\sum_{m=1}^{R} E_{m}$

$\sum_{m=1}^{R} \sum_{j=1}^{R} A_{j m}^{v v} \dot{v}_{j}-\sum_{m=1}^{R} \sum_{j=1}^{R} B_{j m}^{v v} v_{j}-\sum_{m=1}^{G} \sum_{i=1}^{G} C_{i m}^{v p} p_{i}=\sum_{m=1}^{R} E_{m}$

where

$K_{i m}^{p p}=\iint(1-D) \varphi c_{t} P_{i} P_{m} d x d y$

$K_{j m}^{p u}=\iint \frac{\partial P_{m}}{\partial x} N_{j} d x d y$

$K_{j m}^{p v}=\iint \frac{\partial P_{m}}{\partial y} N_{j} d x d y$

$Q_{m}=-\int u_{n} P_{m} d s$

$B_{j m}^{u u}=B_{j m}^{v v}=\iint(1-D) \frac{\mu}{k} N_{j} N_{m} d x d y+\iint D \mu \frac{\partial N_{j}}{\partial x} \frac{\partial N_{m}}{\partial x} d x d y+\iint D \mu \frac{\partial N_{j} \partial N_{m}}{\partial y \partial y} d x d y$

$A_{j m}^{u u}=A_{j m}^{v v}=\iint D \rho N_{j} N_{m} d x d y$

$C_{i m}^{u p}=\iint \frac{\partial P_{i}}{\partial x} N_{m} d x d y$

$C_{i m}^{v p}=\iint \frac{\partial P_{i}}{\partial y} N_{m} d x d y$

$E_{m}=\int D \mu \frac{\partial N_{j}}{\partial n} N_{m} d s$

The matrix form of equations (17)-(19) is: 


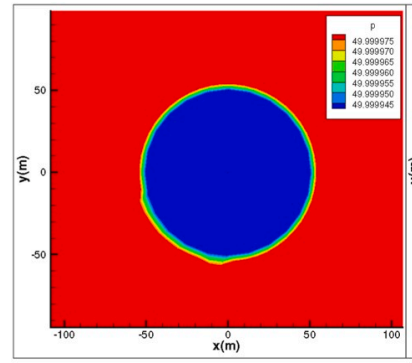

(a) $t=10^{-6} \mathrm{~h}$

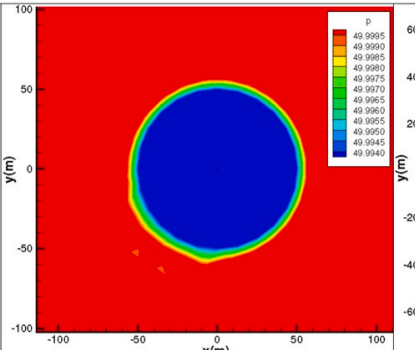

(b) $t=10^{-2} \mathrm{~h}$

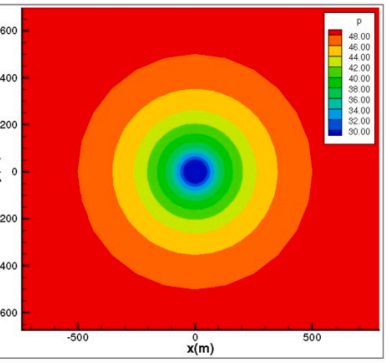

(c) $t=10^{4} \mathrm{~h}$

Fig. 4. Pressure filed of the unfilled WIC model.

Table 1

The input parameters for the unfilled WIC model.

\begin{tabular}{lll}
\hline Parameters & Value & Unit \\
\hline$C$ & 0.001 & $\mathrm{~m}^{3} / \mathrm{MPa}$ \\
$k_{m}$ & 1 & $\mathrm{mD}$ \\
$r_{v}$ & 50 & $\mathrm{~m}$ \\
$\varphi$ & 0.1 & \\
$\mu$ & 1 & $\mathrm{mPa} \mathrm{s}^{-1}$ \\
$c_{t}$ & 0.0035 & $\mathrm{MPa}^{-1}$ \\
$r_{w}$ & 0.1 & $\mathrm{~m}$ \\
\hline
\end{tabular}

$\left[\begin{array}{ccc}K^{p p} & 0 & 0 \\ 0 & A^{u u} & 0 \\ 0 & 0 & A^{v v}\end{array}\right]\left[\begin{array}{c}\dot{p} \\ \dot{u} \\ \dot{v}\end{array}\right]+\left[\begin{array}{ccc}0 & K^{p u} & K^{p v} \\ C^{u p} & B^{u u} & 0 \\ C^{v p} & 0 & B^{v v}\end{array}\right]\left[\begin{array}{l}p \\ u \\ v\end{array}\right]=\left[\begin{array}{c}Q \\ E \\ E\end{array}\right]$

The wellbore boundary and reservoir boundary are the natural boundary conditions, they can be substituted into equation (20). The element stiffness equation (20) are assembled to a global stiffness equation and it is solved by the open source toolkit SuperLU (Li et al., 1999; Li, 2005).

\section{Results and analysis}

\subsection{WIC model}

\subsubsection{Bottom hole pressure response}

Fig. 3 shows bottom hole pressure $p_{w}$ and the derivative $p_{w}$ ' versus $t$ and the input parameters are listed in Table 1 . It can be divided into six stages:

- Stage I: Wellbore storage stage. In this stage, the production is dominated by the compressed fluid in the wellbore while the sand face rate is zero. The pressure and derivative curves are a straight line with unit-slope.

- Stage II: Free-fluid flow stage. With the increase of the sand face rate, the free-fluid flow in the unfilled cavity begins. As can be seen in this stage, the pressure drops sharply and curves oscillate. This is because the free-fluid flow in the unfilled cavity is very fast and it has a good fluid supply for the flow, so the bottom hole pressure decreases in this stage. Fig. 4(a) is the pressure filed of $t=10^{-6} \mathrm{~h}$ which the end time of this stage, it shows that the pressure in the unfilled cavity is almost the same and it can be treated as an equipotential body.

- Stage III: Quasi-wellbore storage flow stage of the cavity. After reaching the boundary of the cavity, the fluid flow transforms from the unfilled cavity to the porous matrix. However, the permeability of the matrix is small, and the unfilled cavity acts like the wellbore. The pressure-drop of the cavity is linear with time, which is called a

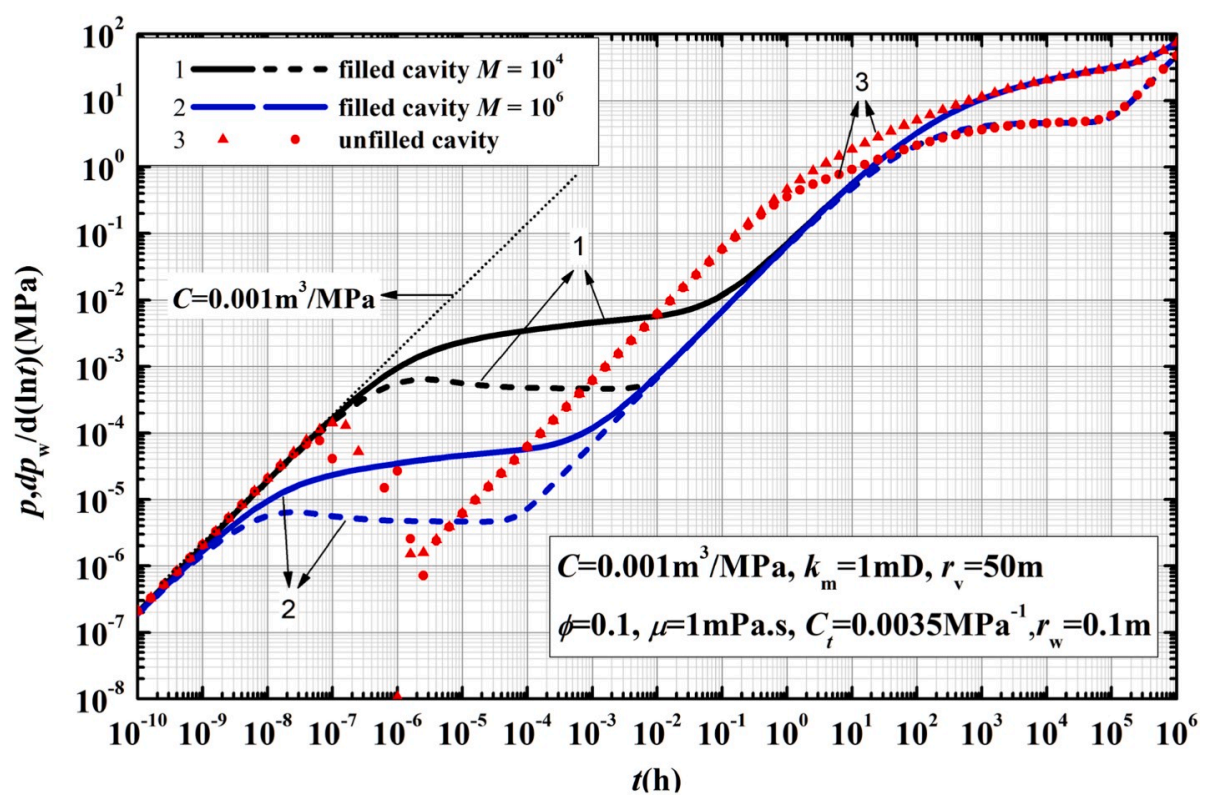

Fig. 5. The comparison of log-log plots between filled WIC model and unfilled WIC model. 


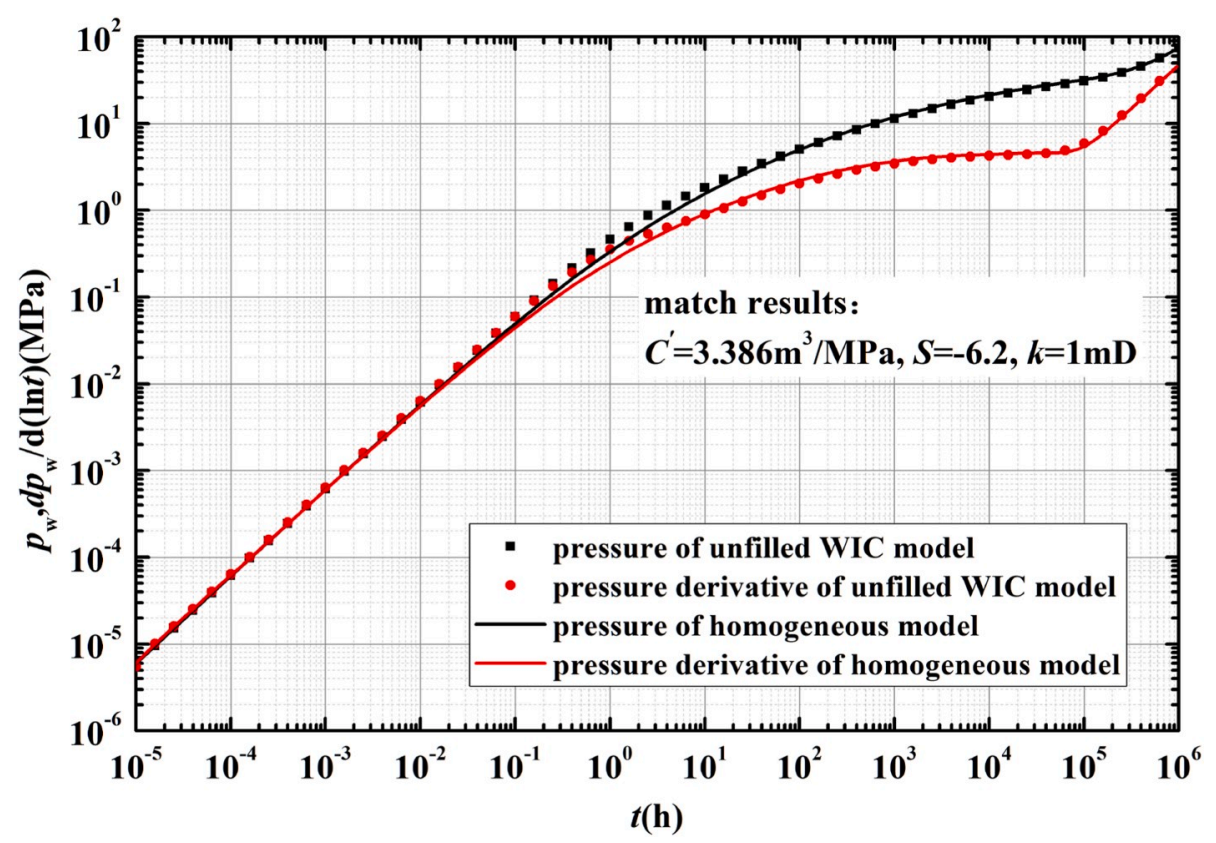

Fig. 6. The matching results of the extracted data with the single-phase Darcy's flow model.

quasi-wellbore storage flow stage. The pressure and derivative are also a straight line with unit-slope in the log-log plot. It can be verified by the pressure field which is presented in Fig. 4(b). In Fig. 4 (b) the pressure-drop is kept in the region of the cavity, but the pressure value is smaller than that in the previous stage.

- Stage IV: Transition stage. With the decrease of the pressure in the cavity, the effect of the fluid in the matrix on the bottom hole pressure is increasing and the flow transits from the cavity to the matrix.

- Stage V: Radial flow stage. After the fluid flow transits to the matrix and the fluid flow gradually develops to radial form which is shown in Fig. 4(c). The derivative curve is a horizontal line in the log-log plot.

- Stage VI: The reservoir boundary response stage. As arriving at the reservoir boundary, the fluid flow has no supply for a closed boundary. Then the bottom hole pressure will increase linearly with time, and pressure derivative is an upward straight line with unitslope.
Fig. 3 shows the type curves of the unfilled WIC model. It should be noticed that the time scale for the wellbore storage stage and the freefluid flow stage is quite small, and limited by the accuracy of pressure gauges, the characteristic of the type curves may not be recognized by the field test data.

\subsubsection{Comparison with the filled cavity}

After analyzing the type curves of the unfilled-cavity model, we also make a comparison with the filled-cavity model. Fig. 5 is the comparative results with the filled model from Wan et al. (2018a). In Fig. 5, the solid lines numbered 1 and 2 are the plots for filled WIC model which the mobility ratio $(M)$ of the cavity is $10^{4}$ and $10^{6}$ which are published in Wan et al. (2018a),'s paper. The dotted lines numbered 3 are the plots for the unfilled WIC model which is shown in Fig. 3. As can be seen, in the wellbore storage stage, the curves of unfilled WIC model and the filled WIC model overlap with each other because the wellbore storage is caused by the fluid compressibility in the wellbore and is independent of the reservoirs. However, in the cavity flow stage, the curves of the two

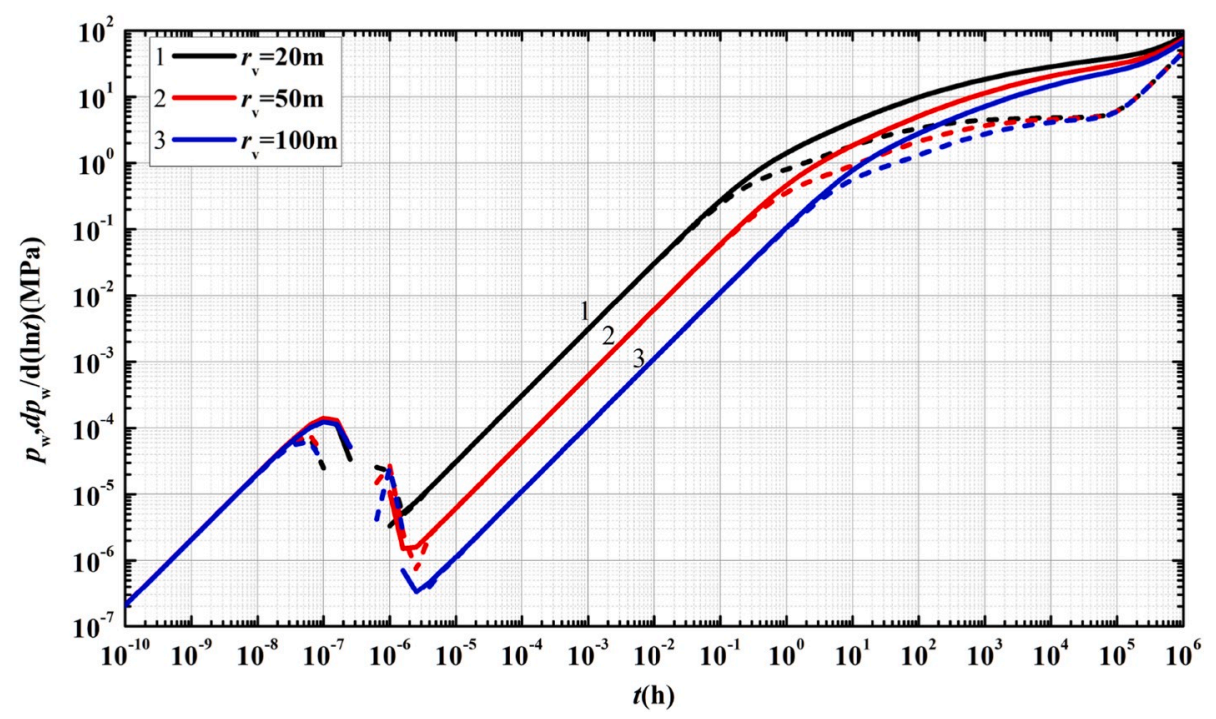

Fig. 7. Log-log plots of the $p_{w}$ and $p_{w}$ 'vs. $t$ for the WIC model with different cavity radius $r_{v}$. 


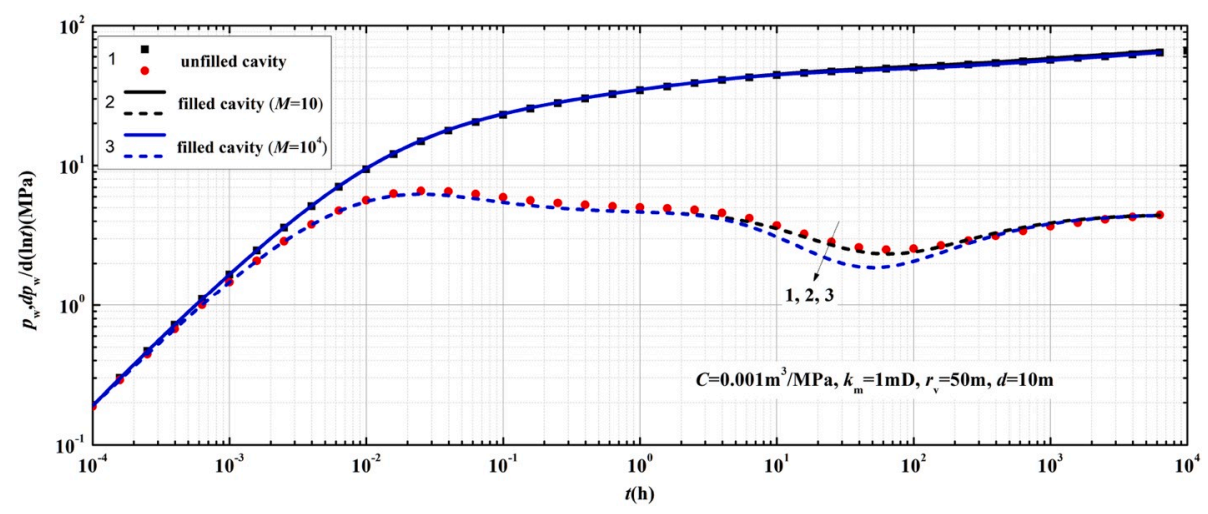

Fig. 8. Log-lot plots of the $p_{w}$ and $p_{w}$ 'vs. $t$ for the filled and unfilled WOC model.

models are quite different. For the filled WIC model, a radial flow of a zero-sloped derivative in the cavity is observed because the cavity is porous media. For the unfilled WIC model, the flow in the cavity is free-fluid flow and a pressure-drop with oscillations rather than a radial flow is found. In the quasi-wellbore storage stage, the straight line of the unfilled WIC model is on the left side of the straight line which represents the transition flow from the cavity to the matrix in the filled WIC model. In the radial flow and reservoir boundary stages, the type curves for filled and unfilled WIC model are exactly the same because the region beyond the cavity are the same.

As analyzed above, the stage iii is the quasi-wellbore storage flow. In this stage, the pressure and derivative curve is an upward straight line with unit-slope and it can be considered as a quasi-wellbore effect. Thus, we extract the data from stage iii to stage vi as a new well test case and used the homogeneous single-phase Darcy's flow model to match the extracted data. Fig. 6 is the matching result. The matched permeability of the matrix is exactly the same as the unfilled WIC model. The matched wellbore storage $C^{\prime}$ is $3.386 \mathrm{~m}^{3} / \mathrm{MPa}$ and the skin factor $S$ is -6.2 . As mentioned above, the unfilled cavity can be treated as wellbore and thus the radius of the cavity is the effective wellbore radius. Then, we use the effective wellbore radius model to compute the skin factor. We get,

$r_{v}=r_{w} e^{-S}$

The radius of the cavity is $r_{v}=50 \mathrm{~m}$ and the radius of the wellbore is $r_{w}=0.1 \mathrm{~m}$, substituting into equation (21) to compute the skin factor,

$S=-\ln \frac{r_{v}}{r_{w}}=-\ln \frac{50}{0.1}=-6.21$

and results are the same with the matched result from Fig. 6. This verifies that it is feasible to treat the unfilled cavity as a wellbore and as a consequence the wellbore storage coefficient increases. This explained why a large wellbore storage and negative skin factor are observed in the field test of the caved carbonated reservoirs.

\subsubsection{Influence of the cavity radius $r_{v}$}

Fig. 7 is the log-log plots of the $p_{w}$ and $p_{w}$ ' vs. $t$ with different cavity radius $r_{v}$. As can be seen, the upward straight line in the stage iii is shifted to the right when the $r_{v}$ increases, which indicates that the quasiwellbore storage coefficient increase with the cavity radius.

\subsection{WOC model}

\subsubsection{Bottom hole pressure response and comparison with filled WOC model}

Fig. 8 is the $\log -\log$ plots of the $p_{w}$ and $p_{w}$ ' versus $t$ for the unfilled WOC model (curves numbered 1) and the filled WIC model (curves numbered 2 and 3). As Wan et al. (2018a), analyzed, the characteristic of the type curves for the WOC model is the valley on the derivative, which is caused by the high permeability of the cavity. As can be seen from Fig. 8, the type curves of the unfilled WOC model are very similar to the unfilled WOC model. It is because the cavity is a good fluid supply for the wellbore no matter it is filled or unfilled. The depth of the valley is dependent on the mobility ratio of the cavity $M$ which determines the fluid mobility in the cavity. The greater $M$, the deeper the valley, but there is a limit of the depth, which is believed to be the unfilled cavity. However, from Fig. 8, we can see that the depth of the unfilled cavity is smaller than the filled cavity. We believe it is because the fluid flow in the cavity is assumed to be incompressible and the contribution of the fluid compressibility to the valley is neglected.

\subsubsection{Influence of the cavity radius $r_{v}$}

Fig. 9 is the log-log plots of the WOC model with different cavity radius $r_{v}$, which could be $30 \mathrm{~m}, 50 \mathrm{~m}$, and $100 \mathrm{~m}$. As can be seen, the larger the cavity is, the deeper the valley on the derivative, which is the same with the filled WOC model (Wan et al., 2018a). In Fig. 9, we can see that the valley on the derivative is not very deep even if the radius of the cavity is as much as $100 \mathrm{~m}$. It means that the influence of the cavity

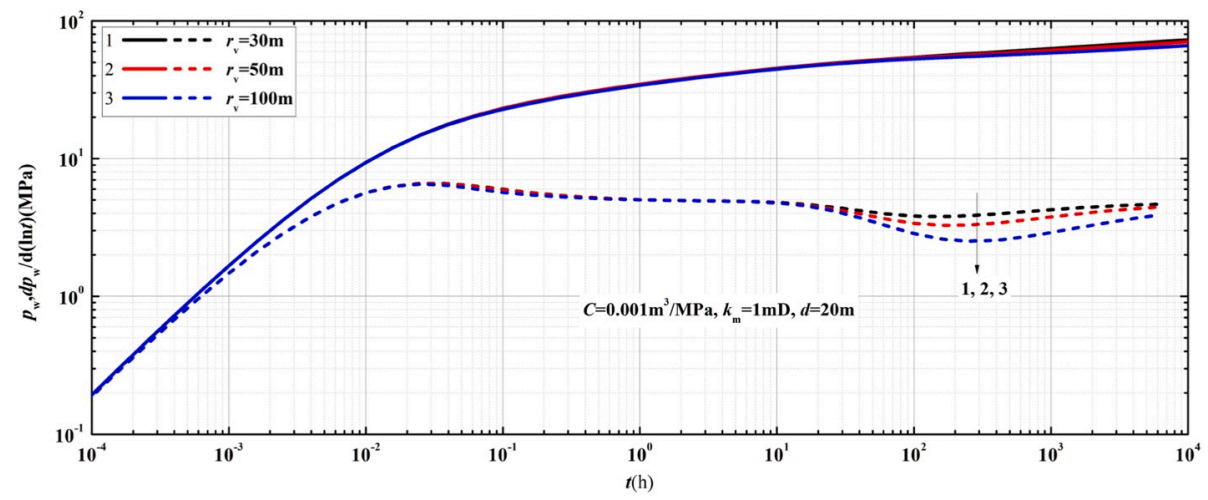

Fig. 9. Log-log plots of the $p_{w}$ and $p_{w}$ ' vs. $t$ for the WOC model with different $r_{v}$. 


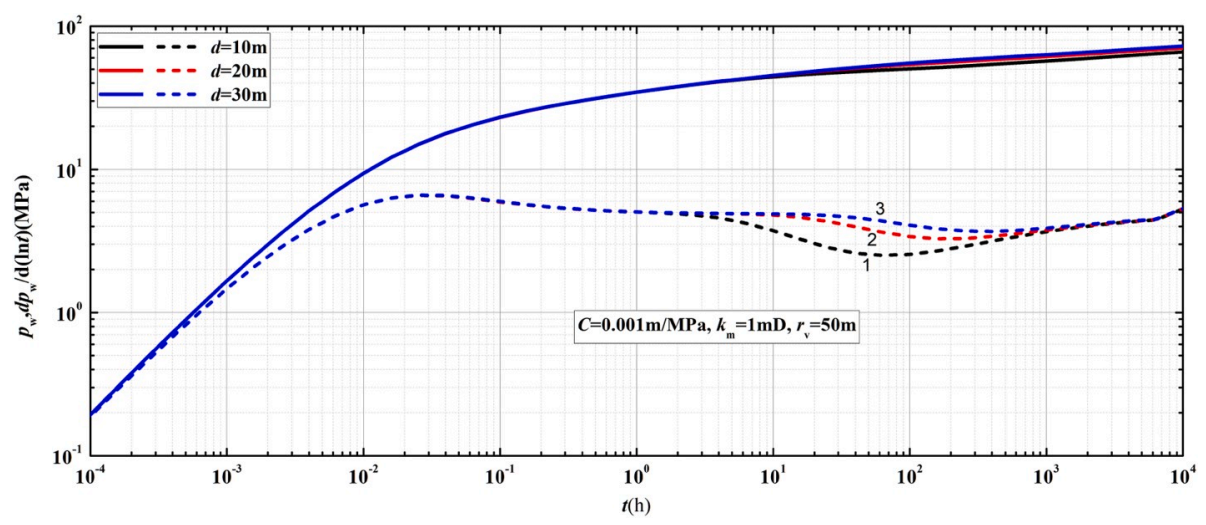

Fig. 10. Log-log plots of the $p_{w}$ and $p_{w}$ ' vs.t for the WOC model with different $d$.

on the pressure derivative in the case of WOC is quite small compared with the case of WIC.

\subsubsection{Influence of the distance $d$ from the cavity to the wellbore}

Fig. 10 is the log-log plots for the WOC model with different cavity position. The curves numbered 1,2 , and 3 are the cases of the $d$ is $10 \mathrm{~m}$, $20 \mathrm{~m}$, and $30 \mathrm{~m}$, respectively. As can be seen, the farther the cavity is from the wellbore, the later the valley begins and the smaller the depth of the valley on the derivative, which is also the same the filled WOC model. From Fig. 10, we can see that the influence of the cavity is obvious only when it is close to the wellbore. On the other hand, the cavities far from the wellbore can be ignored in the well test interpretation.

\section{Conclusion}

This paper develops a numerical pressure transient model based on Stokes-Darcy coupled theory for the caved-carbonate reservoirs with unfilled cavities, in which the flow is described by Stokes' equation. The finite element method is applied in the solutions of the mathematical models. The type curves of two typical cases of a well drilled into the cavity and not drilled into the cavity are analyzed. Conclusions can be drawn as followings:

- The type curves of the well drilled into the unfilled cavity model can be divided into six stages. The stages dominated by the unfilled cavity are the free-fluid flow stage and the quasi-wellbore storage flow stage.

- Compared with the filled cavity model presented in the previous work, in the free-fluid flow stage, a pressure-drop with oscillations rather than a radial flow is observed. After the free-fluid flow stage, a long duration of quasi-wellbore storage stage is found in which the unfilled cavity is an equipotential body.

\section{Nomenclature}

$d$ distance between the center of the cavity and the wellbore, $\mathrm{L}$

$r_{v} \quad$ radius of cavity, $\mathrm{L}$

$\varphi \quad$ porosity

$c_{t} \quad$ total compressibility coefficient, $\mathrm{M}^{-1} \mathrm{LT}^{2}$

$p \quad$ pressure, $\mathrm{ML}^{-1} \mathrm{LT}^{2}$

$t$ time, $\mathrm{T}$

$u \quad$ fluid velocity vector, $\mathrm{LT}^{-1}$

$u \quad$ fluid velocity in $x$ direction, $\mathrm{LT}^{-1}$

$v \quad$ fluid velocity in $y$ direction, $\mathrm{LT}^{-1}$

$k \quad$ permeability, $\mathrm{L}^{2}$

$\mu \quad$ fluid viscosity, $\mathrm{ML}^{-1} \mathrm{~T}^{-1}$
- An unfilled cavity can be considered as an enlarged wellbore which can be equivalent to a negative skin factor and a larger wellbore storage coefficient would be obtained.

- The type curves of the well not drilled into the unfilled cavity have a valley on the derivative caused by the cavity. They are very similar to the filled cavity model. However, the unfilled cavity model is not the limit case of the filled cavity model with extremely large mobility of the cavity.

- A cavity with a larger size and smaller distance from the wellbore would give rise to a deeper valley.

\section{Declaration of competing interest}

The authors declare that they have no known competing financial interests or personal relationships that could have appeared to influence the work reported in this paper.

\section{CRediT authorship contribution statement}

Yizhao Wan: Conceptualization, Methodology, Software, Validation, Formal analysis, Writing - original draft, Writing - review \& editing, Visualization. Yuewu Liu: Resources, Project administration. Nengyou Wu: Resources, Writing - review \& editing.

\section{Acknowledgments}

This work was supported by the National Natural Science Foundation of China, grant No. 41906187, the Laboratory for Marine Mineral Resources, Qingdao National Laboratory for Marine Science and Technology, China, grant No. MMRZZ201807, Shandong Provincial Natural Science Foundation, China, grant No. ZR2019BD058, and Taishan Scholar Special Experts, China Project (No: ts201712079). 
$\rho \quad$ fluid density, $\mathrm{ML}^{-3}$

D model control coefficient

$r_{\mathrm{w}} \quad$ radius of wellbore, $\mathrm{L}$

$\Gamma \quad$ boundary

$q \quad$ fluid volume rate, $\mathrm{L}^{3} \mathrm{~T}^{-1}$

$B \quad$ volume factor of the fluid

C wellbore storage coefficient

$P \quad$ interpolation function of pressure

$N \quad$ interpolation function of velocity

$G \quad$ number of the interpolation points for $p$

$R \quad$ number of the interpolation points for $u$ and $v$

$G \quad$ number of the interpolation points for $p$

$\begin{array}{ll}\text { Subscripts } \\ \mathrm{m} & \text { matrix } \\ \mathrm{c} & \text { cavity } \\ \mathrm{w} & \text { wellbore }\end{array}$

Appendix A. Supplementary data

Supplementary data to this article can be found online at https://doi.org/10.1016/j.petrol.2020.107085.

\section{References}

Arbogast, T., Gomez, M.S.M., 2009. A discretization and multigrid solver for a Darcy-Stokes system of three dimensional vuggy porous media. Comput. Geosci. 13 (3), 331-348.

Beavers, G.S., Joseph, D.D., 1967. Boundary conditions at a naturally permeable wall. J. Fluid Mech. 30 (1), 197-207.

Beavers, G.S., Sparrow, E.M., Magnuson, R.A., 1970. Experiments on coupled parallel flows in a channel and a bounding porous medium. J. Basic Eng. 92 (4), 843-848.

Chen, P., Wang, X., Liu, H., Huang, Y., Chen, S., Zhang, H., 2016. A pressure-transient model for a fractured-vuggy carbonate reservoir with large-scale cave. Geosystem Eng. 19 (2), 69-76.

Deng, C., Martinez, D., 2005. Viscous flow in a channel partially filled with a porous medium and with wall suction. Chem. Eng. Sci. 60 (2), 329-336.

Djatmiko, W., Hansamuit, V., et al., 2010. Well test analysis of multiple matrix-tofracture fluid transfer in fractured-vuggy reservoir. In: International Oil and Gas Conference and Exhibition in China. Society of Petroleum Engineers.

Gao, B., Huang, Z.-Q., Yao, J., Lv, X.-R., Wu, Y.-S., 2016. Pressure transient analysis of a well penetrating a filled cavity in naturally fractured carbonate reservoirs. J. Petrol. Sci. Eng. 145, 392-403.

Gomez, S., Camacho, R., Vasquez, M., Ramos, G., del Castillo, N., Mesejo, J., et al., 2014. Well test characterization of naturally fractured vuggy reservoirs, with a global optimization method. In: Offshore Technology Conference-Asia, Offshore Technology Conference.

Gulbransen, A.F., Hauge, V.L., Lie, K.-A., et al., 2010. A multiscale mixed finite element method for vuggy and naturally fractured reservoirs. SPE J. 15 (2), 395-403.

Hu, X., Quan, L., Qi, D., 2014. Features of cavern filling in fractured/vuggy carbonate oil reservoirs, tahe oilfield. Special Oil Gas Reservoirs 21 (1), 18-21.

Huang, Z., 2012. Theoretical Study on Multiscale Modeling of Two-phase Flow Based on Discrete Fracture-Vug Model. Ph.D. thesis. China University of Petroleum (EastChina).

Huang, Z., Yao, J., Li, Y., Wang, C., Lv, X., 2011. Numerical calculation of equivalent permeability tensor for fractured vuggy porous media based on homogenization theory. Commun. Comput. Phys. 9 (1), 180-204.

Huang, Z.-Q., Yao, J., Wang, Y.-Y., 2013. An efficient numerical model for immiscible two-phase flow in fractured karst reservoirs. Commun. Comput. Phys. 13 (2), 540-558.

Jiang, R., Zhang, C., Cui, Y., Wang, Q., Zhang, W., Zhang, F., 2019. Characteristics of transient pressure performance of horizontal wells in fractured-vuggy tight fractal reservoirs considering nonlinear seepage. Oil and Gas Sci. Technol. - Revue d'IFP Energies Nouvelles 74, 57.

Li, X.S., 2005. An overview of SuperLU: algorithms, implementation, and user interface. ACM Trans, Math Software 31 (3), 302-325.

Li, X., Demmel, J., Gilbert, J., Grigori, iL., Shao, M., Yamazaki, I., September 1999. SuperLU Users' Guide, Tech. Rep. LBNL-44289. Lawrence Berkeley National Laboratory. http://crd.lbl.gov/ xiaoye/SuperLU/. (Accessed August 2011).

Li, Y., Zhang, J., Liu, Z., Li, B., Deng, X., et al., 2016. A systematic technique of production forecast for fractured vuggy carbonate gas condensate reservoirs. In: SPE Kingdom of Saudi Arabia Annual Technical Symposium and Exhibition. Society of Petroleum Engineers.

Li, Y., Kand, Z., Xue, Z., Zheng, S., 2018. Theories and practices of carbonate reservoirs development in China. Petrol. Explor. Dev. 45 (4), 712-722.

Liu, H., Wang, X., 2012. Pressure response characteristics in large scale cavity type reservoir. J. Southwest Pet. Univ. (Sci. Technol. Ed.) 34 (4), 94-99.
Mikelic, A., Jäger, W., 2000. On the interface boundary condition of beavers, joseph, and saffman. SIAM J. Appl. Math. 60 (4), 1111-1127.

Peng, X., Du, Z., Liang, B., Qi, Z., et al., 2009. Darcy-Stokes streamline simulation for the tahe-fractured reservoir with cavities. SPE J. 14 (3), 543-552.

Popov, P., Qin, G., Bi, L., Efendiev, Y., Ewing, R.E., Li, J., et al., 2009. Multiphysics and multiscale methods for modeling fluid flow through naturally fractured carbonate karst reservoirs. SPE Reservoir Eval. Eng. 12 (2), 218-231.

Raghavan, R., Chen, C., 2019. The theis solution for subdiffusive flow in rocks. Oil Gas Sci. Technol. 74 (3), 6.

Saffman, P.G., 1971. On the boundary condition at the surface of a porous medium. Stud. Appl. Math. 50 (2), 93-101.

Wan, Y., Liu, Y., 2016. Numerical investigation of dual-porosity model with transient transfer function based on discrete-fracture model. Appl. Math. Mech. 37 (5), 611-626.

Wan, Y., Liu, Y., Liu, W., Han, G., Niu, C., 2016. A numerical approach for pressure transient analysis of a vertical well with complex fractures. Acta Mech. Sin. 32 (4), 640-648.

Wan, Y.-Z., Liu, Y.-W., Chen, F.-F., Wu, N.-Y., Hu, G.-W., 2018a. Numerical well test model for caved carbonate reservoirs and its application in tarim basin, China. J. Petrol. Sci. Eng. 161, 611-624.

Wan, Y., Liu, Y., Wu, N., Hu, G., 2018b. A numerical well test model for multi-fractured horizontal wells based on discrete-fracture model and its application. Chin. J. Theor. Appl. Mech. 50 (1), 147-156.

Wan, Y., Wu, N., Liu, C., Chen, Q., Liu, Y., 2019. 3d pressure transient analysis model of fractured-caved reservoirs based on seismic characterization. J. Porous Media 22 (11), 1351-1370.

Wu, Y.-S., Ehlig-Economides, C., Qin, G., Kang, Z., Zhang, W., Ajayi, B., Tao, Q., 2007. A Triple-Continuum Pressure-Transient Model for a Naturallyfractured Vuggy Reservoir, Tech. Rep. Ernest Orlando Lawrence Berkeley NationalLaboratory, Berkeley, CA (US).

Wu, Y.-S., Di, Y., Kang, Z., Fakcharoenphol, P., 2011. A multiple-continuum model for simulating single-phase and multiphase flow in naturally fractured vuggy reservoirs. J. Petrol. Sci. Eng. 78 (1), 13-22.

Xiao, C., Liu, X., Zhang, C., Yang, P., Dan, G., Li, X., et al., 2010. Characterizing fractured-vuggy carbonate reservoirs quantitatively based on the integration of well and seismic. In: SPWLA 51st Annual Logging Symposium. Society of Petrophysicists and Well-Log Analysts.

Xing, C., Yin, H., Liu, K., Li, X., Fu, J., 2018. Well test analysis for fractured and vuggy carbonate reservoirs of well drilling in large scale cave. Energies 11 (1), 80.

Yao, J., Huang, Z., Wang, Z., Li, Y., Wang, C., 2010. Mathematical model of fluid flow in fractured vuggy reservoirs based on discrete fracture-vug network. Acta Pet. Sin. 31 (5), 815-819+824.

Zhang, F., Chen, F., Peng, J., Jia, Y., Lei, S., Yang, X., Jianjun, Y., 2009. A well test model for wells drilled in big-size cavity of naturally fractured vuggy carbonate reservoirs. Acta Pet. Sin. 6 (30), 912-915.

Zhang, W., Jin, Q., Xu, S., Tian, F., Cui, J., 2012. Paleo- cavern filling characteristics and hydrocarbon reservoir implication in the ordovician outcrops in northern tarim basin. Tezhong Youqicang- Special Oil \& Gas Reservoirs 19 (3), 50-54.

Zhao, W., Shen, A., Qiao, Z., Zheng, J., Wang, X., 2014. Carbonate karst reservoirs of the tarim basin, northwest China: types, features, origins, and implications for hydrocarbon exploration. Interpretation 2 (3), SF65-SF90.

Zhu, G., Zou, C., Yang, H., Wang, K., Zheng, D., Zhu, Y., Wang, Y., 2015. Hydrocarbon accumulation mechanisms and industrial exploration depth of large-area fracture-cavity carbonates in the tarim basin, western China. J. Petrol. Sci. Eng. 133, 889-907. 